\title{
Estrogen protects cardiomyocytes against lipopolysaccharide by inhibiting autophagy
}

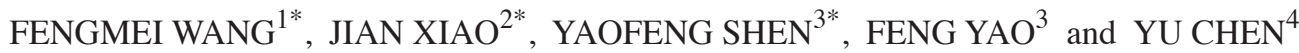 \\ ${ }^{1}$ Department of Obstetrics and Gynaecology, Fuzhou General Hospital, Fuzhou, Fujian 350025; ${ }^{2}$ Department of \\ Cardiothoracic Surgery, Changzheng Hospital, The Second Military Medical University, Shanghai 200003; \\ ${ }^{3}$ Department of Anesthesiology, Shanghai Chest Hospital Affiliated to Shanghai Jiaotong University, \\ Shanghai 200030; ${ }^{4}$ Department of Obstetrics and Gynaecology, Changhai Hospital, \\ The Second Military Medical University, Shanghai 200433, P.R. China
}

Received October 10,2013; Accepted March 27, 2014

DOI: $10.3892 / \mathrm{mmr} .2014 .2365$

\begin{abstract}
Autophagy has a significant role in myocardial injury induced by lipopolysaccharide (LPS). Estrogen (E2) has been demonstrated to protect cardiomyocytes against apoptosis; however, it remains to be determined whether it exhibits anti-autophagic effects. The aim of the present study was to investigate whether estrogen-regulated autophagy attenuates cardiomyocyte injury induced by LPS. The cardiomyocytes of neonatal rats were randomized to the control (Con), LPS and estrogen + LPS groups. The LPS group was treated with $1 \mu \mathrm{g}$ LPS for $24 \mathrm{~h}$ and the estrogen + LPS group was treated with $10^{-8} \mathrm{M}$ estrogen $30 \mathrm{~min}$ prior to treatment with LPS. Cardiomyocyte autophagy was quantitated by investigating the mRNA and protein level of autophagy-related genes (Atgs). The mRNA expression of Atg5 and Beclin1 were measured by quantitative polymerase chain reaction and the microtubule-associated protein light chain 3 (LC3) protein expression was measured by western blot analysis. To demonstrate the cardiomyocyte protection of estrogen, cell vitality and serum lactate dehydrogenase (LDH) levels were measured following LPS treatment. It was identified that LPS induced cardiomyocyte injury, together with the upregulation of Atg5, Beclin1 mRNA and LC3-II protein. Furthermore, estrogen attenuated the effect of LPS. The present study provides evidence that estrogen has a myocardial protective role against injury induced by LPS by regulating autophagy.
\end{abstract}

Correspondence to: Dr Yu Chen, Department of Obstetrics and Gynaecology, Changhai Hospital, The Second Military Medical University, 172 Changhai Road, Shanghai 200433, P.R. China

E-mail: yuchen1979@outlook.com

*Contributed equally

Key words: estrogen, cardiomyocyte, autophagy, lipopolysaccharide, microtubule-associated protein light chain 3

\section{Introduction}

The myocardium is one of the target organs of septic shock (1-2), which is a major cause of mortality. This myocardial injury may occur as a result of the release of proinflammatory cytokines induced by bacterial endotoxin lipopolysaccharide (LPS) (3). Furthermore, it appears that LPS may be responsible for multiple organ failure during septic shock (4). It has also been demonstrated that LPS reduced myocardial function (5). Numerous studies have suggested that LPS-induced myocardial dysfunction is mediated by multiple proinflammatory mediators, including tumor necrosis factor- $\alpha$ (TNF- $\alpha$ ), Toll-like receptor 4 (TLR4) and TLR2 (6-8).

Recently, LPS was reported to stimulate cardiomyocyte autophagy $(4,9)$, which may mediate cell death. Autophagy, which has been suggested to be an essential function for cell homeostasis, as well as cell defense and adaptation to an adverse environment, is a type of programmed cell death (10-12). Autophagy has an important role in the heart, and activation of autophagy has been observed in a variety of heart diseases, including cardiac hypertrophy, heart failure and ischemia reperfusion injury. Therefore, it is important to regulate cardiomyocyte autophagy in order to reduce myocardial injury induced by LPS.

It is well established that the incidence of cardiovascular disease is reduced in females prior to menopause, which may be due to estrogen (E2) levels (13-14). Studies have demonstrated that E2 exhibits cardioprotective effects due to the ability to decrease TNF- $\alpha$ levels (15). However, few studies have investigated whether E2 may regulate cardiomyocyte autophagy. Based on these observations, the present study aimed to examine whether E2 may reduce cardiomyocyte injury by regulating autophagy.

\section{Materials and methods}

Animal care. All animal experiments were approved by the Animal Research Ethics Committee of the Second Military Medical University (Shanghai, China). The experimental procedures conformed with the guide for the care and use 
of laboratory animals published by the US National Institutes of Health.

Cell culture and experimental procedures. Neonatal cardiomyocytes were prepared from the hearts of Sprague-Dawley rats younger than 3 days (16). On the 4 th day, the cardiomyocytes were randomized to three groups: The control group (con), where the cells were cultured in Dulbecco's modified Eagle's medium (DMEM) with $5 \% \mathrm{CO}_{2}$ and $95 \%$ air for $24 \mathrm{~h}$; the LPS group, where the cells were treated with $1 \mu \mathrm{g} / \mathrm{ml} \mathrm{LPS} \mathrm{for} 24 \mathrm{~h}$; and the E2+LPS group, where the cells were treated with $10^{-8} \mathrm{M}$ $\mathrm{E} 2$, and then were treated with $1 \mu \mathrm{g} / \mathrm{ml}$ LPS 30 min later.

\section{3-(4,5-dimethylthiazol-2-yl)-2,5-diphenyl tetrazonium bromide} (MTT) assay. For the MTT assay, $10 \mu \mathrm{l}$ MTT solution was added to the growing cells and incubated for $4 \mathrm{~h}$. The crystals were then solubilized by adding $100 \mu$ l solubilization solution. The absorbance of the purple solution was determined at a wavelength of $450 \mathrm{~nm}$ with a microtiter plate reader (Bio-Rad, Hercules, CA, USA).

Lactate dehydrogenase $(L D H)$ assay. $\mathrm{LDH}$ release was measured following treatment as a cellular injury index. The culture media was collected for determination of LDH activity using an Hitachi 7020 chemistry analyzer (Hitachi, Ltd., Tokyo, Japan).

Quantitative polymerase chain reaction ( $q P C R$ ) of Atg5 and Beclin1. Total RNA of cells was isolated using TRIzol reagent and reverse transcribed according to the manufacturer's instructions (Thermo Scientific, Waltham, MA, USA). Dysregulated Atg5 and Beclin1 were validated by qPCR in duplicates using the Mini OPTICON realtime PCR system (Bio-Rad). The annealing temperature of Atg5 and Beclin1 was set at $56^{\circ} \mathrm{C}$. The comparative $\mathrm{Ct}$ (threshold cycle) method with arithmetic formulae $\left(2^{-\Delta \Delta \mathrm{Ct}}\right)$ was used to determine the relative quantitation of gene expression of the target and housekeeping genes ( $\beta$-actin). The following sense and antisense primers were used: Beclin1 (accession number NM_001034117), forward 5'-GGCAGTGGCTCCTATT-3' and reverse 5'-GGCGTGCTGTGCTCTGAAAA-3'; Atg5(accessionnumber NM_001014250), forward 5'-AGTGGAGGCAACAGAACC-3' and reverse 5'-GACACGAACTGGCACATT-3'.

Western blotting of microtubule-associated protein light chain 3 (LC3). The protein concentration was determined with a bicinchoninic acid protein assay kit (Beyotime Institute of Biotechnology, Haimen, Jiangsu, China) according the manufacturer's instructions. Equal quantities of protein $(40 \mu \mathrm{g})$ from the cardiomyocytes were subjected to western blotting analysis to evaluate LC3 expression (the primary rabbit antibody was purchased from Sigma, St. Louis, MO, USA) with an enhanced chemiluminescence detection kit (Amersham Biosciences, Piscataway, NJ, USA). The results are presented as LC3-II/LC3-I.

Statistical analysis. Quantitative data are presented as the mean \pm standard error. Statistical significance was determined using one-way analysis of variance. $\mathrm{P}<0.05$ was considered to indicate a statistically significant difference.

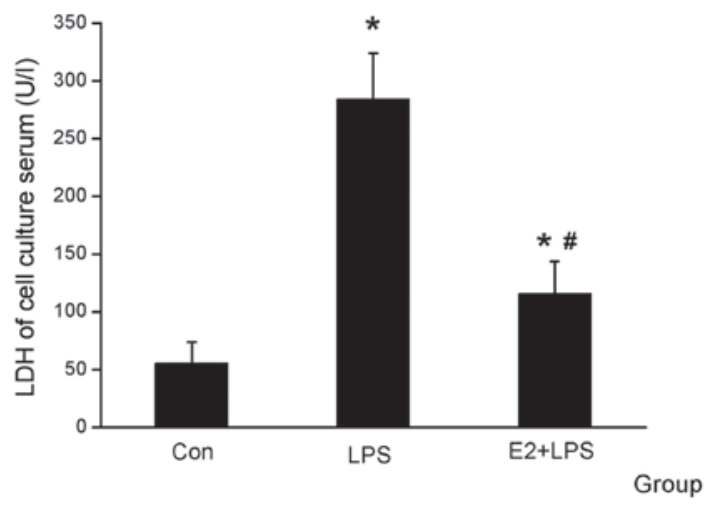

Figure 1. Results of LDH in the cell culture serum $(n=6)$. It was identified that LDH was increased following treatment with LPS $\left({ }^{*} \mathrm{P}<0.05\right)$ and was decreased in the E2 + LPS group compared with the LPS group $\left({ }^{\#} \mathrm{P}<0.05\right)$. LDH, lactate dehydrogenase; LPS, lipopolysaccharide; E2, estrogen; Con, control.

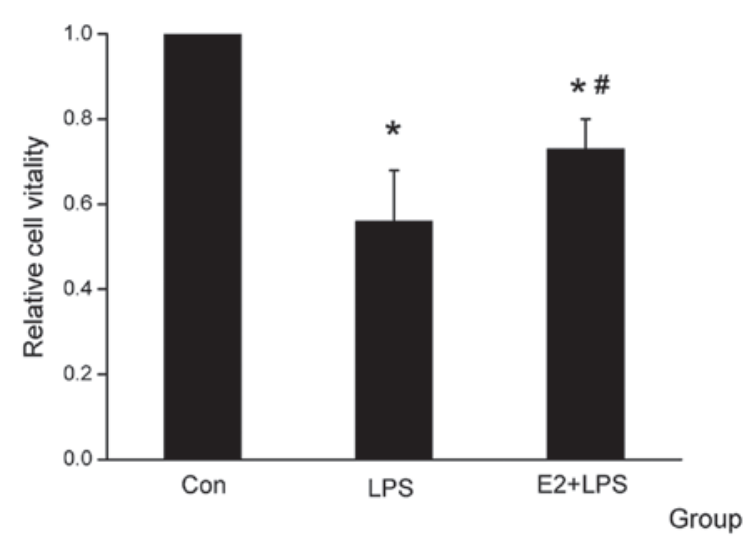

Figure 2. Results of relative cell vitality $(n=6)$. It was identified that cell vitality was decreased following treatment with LPS $\left({ }^{*} \mathrm{P}<0.05\right)$, and was increased in the E2 + LPS group compared with the LPS group $\left({ }^{\#} \mathrm{P}<0.05\right)$. E2, estrogen; LPS, lipopolysaccharide; Con, control.

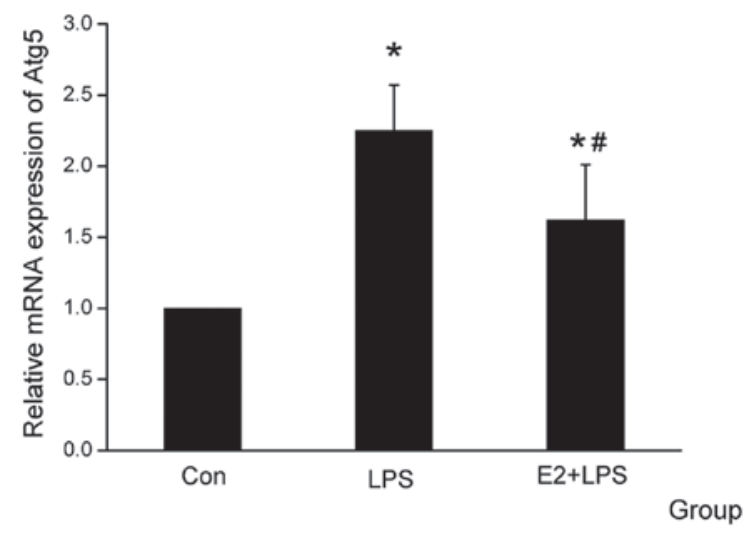

Figure 3. Results of Atg 5 expression with quantitative polymerase chain reaction $(n=5)$. Atg5 was upregulated by LPS compared with the Con group $\left({ }^{*} \mathrm{P}<0.05\right)$ and was downregulated by E2 compared with the LPS group $\left({ }^{\#} \mathrm{P}<0.05\right)$. LPS, lipopolysaccharide; E2, estrogen; Con, control.

\section{Results}

E2 produces a cardioprotective effect against LPS. The serum was collected for the LDH and MTT assays and was used for 


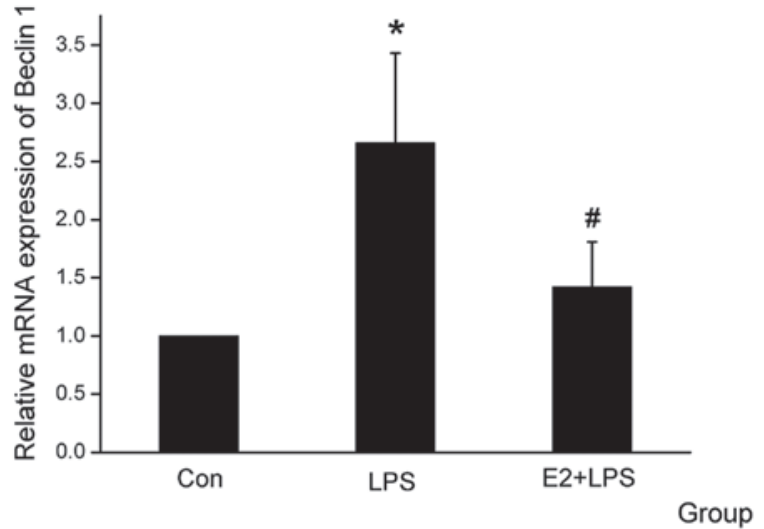

Figure 4. Results of Beclin1 expression with quantitative polymerase chain reaction $(n=5)$. Beclin1 was upregulated by LPS compared with the Con group $\left({ }^{*} \mathrm{P}<0.05\right)$, while was downregulated by E2 compared with the LPS group $\left({ }^{\#} \mathrm{P}<0.05\right)$. E2, estrogen; LPS, lipopolysaccharide; Con, control.

A

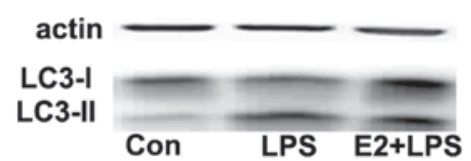

B

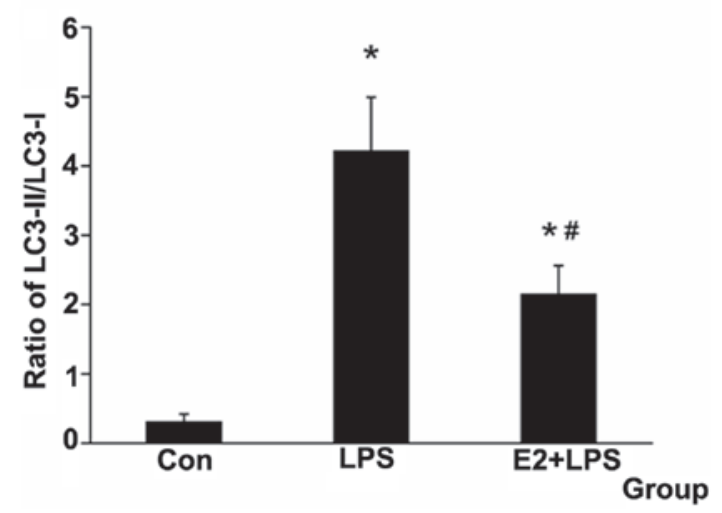

Figure 5. Results of LC3 protein expression with western blotting $(n=5)$. (A) Representative western blot of LC3 from the different groups. (B) The ratio of LC3-II/LC3-I in different groups. It was identified that LC3-II was upregulated by LPS $(" \mathrm{P}<0.05)$ and it was attenuated by E2 $\left({ }^{*} \mathrm{P}<0.05\right)$. LC3, microtubule-associated protein light chain 3; E2, estrogen; LPS, lipopolysaccharide; Con, control.

comparing cardiomyocyte vitality. It was identified that LDH was higher and the cell vitality was decreased in the LPS group compared with the Con group, and that E2 was able to attenuate the effect of LPS (Figs. 1 and 2).

E2 attenuates Atg5 and Beclin1 mRNA expression level. The Atg family members, particularly Beclin1 and Atg5, have been reported to have an important role in the autophagic cell death pathway (17-18). When the cardiomyocytes were treated with E2, the mRNA expression of Atg5 and Beclin1 was downregulated, which was upregulated by LPS (Figs. 3 and 4).

E2 attenuates the LC3-II protein relative expression level. LC3-II was used as a marker of autophagy (19). The ratio of LC3-II/LC3-I was used to examine the autophagy level in the present study. It was identified that the ratio was increased in the LPS group and decreased in the E2 + LPS group (Fig. 5).

\section{Discussion}

LPS, which is a major component of bacterial outer walls, has been demonstrated to be responsible for the multiorgan dysfunction that characterizes septic shock (4). It has been found that LPS is able to stimulate inflammatory mediator production and activate $\mathrm{NF}-\kappa \mathrm{B}(20-21)$. The myocardium is one of the main target organs of septic shock (2). Therefore, it is clinically beneficial to investigate ways to attenuate the myocardial injury induced by LPS in patients with septic shock. In the present study LPS was used to simulate the heart injury induced by septic shock in vitro. According to this model, the present study aimed to investigate novel reagents that may protect the heart against LPS injury. LDH and cell vitality were generally used to evaluate cell injury $(16,22)$. It was found that LPS was harmful to the cardiomyocytes and E2 attenuated the injury induced by LPS.

Apoptosis, necrosis and autophagy occur in cardiomyocytes during cardiac injury (23). The autophagy process is regulated by Atgs, among which Beclin1 is required for the autophagy vesicle nucleation step of autophagy. The autophagosome is formatted through two pathways, the Atg12-Atg5-Atg16 pathway and the Atg4-Atg7-Atg3 pathway. These conjugations lead to the conversion of the soluble form of LC3 (LC3-I) to the autophagic vesicle-associated form (LC3-II), which is used as a marker of autophagy (19). In the majority of these studies, the ratio of LC3-II/LC3-I has been used for examining the autophagy level (24).

Apoptosis and necrosis are well established as detrimental processes to the heart (25). However, the effect of autophagy in the heart is controversial (26-27). At low levels, autophagy removed the damaged proteins and organelles, that facilitated myocardial survival during periods of energy deprivation (28). Therefore, low levels of autophagy are beneficial to cardiomyocytes (29). However, excessive levels of autophagy appear to contribute to cardiomyocyte damage (24). Furthermore, accumulation of autophagic vacuoles precedes apoptotic cell death, and autophagy induces cell death when apoptosis is inhibited. For instance, it has been demonstrated that autophagy was marginally increased in the myocardium during the ischemic period, and it was protective for the heart, while during the reperfusion period autophagy was markedly enhanced and was subsequently harmful to the heart $(24,27)$. Furthermore, it has been identified that the inhibition of autophagy is cardioprotective against LPS-induced injury (9). Therefore, moderate regulation of autophagy may aid in attenuating cardiomyocyte injury induced by LPS.

Studies have suggested that E2 has important cardioprotective roles against ischemia-reperfusion (IR) injury (30-31) and that E2 treatment may upregulate the expression of anti-apoptotic genes (32). Recently, several studies demonstrated that Beclin1 was able to downregulate E2ic signaling, suggesting the importance of the interaction between E2 and autophagy (33).

The biological effects of E2 are predominantly mediated via E2 receptors (ERs). The two classic ER isoforms (ER $\alpha$ and $\mathrm{ER} \beta)$ are expressed in cardiomyocytes, and there appears to be no difference in the distribution or abundance between males 
and females (30). ER $\alpha$ is mainly expressed in the ventricles and its activation may result in rapid cardioprotective signaling (34). $\mathrm{ER} \beta$ is evenly distributed throughout the heart and may not be involved in cardioprotection (35-36). E2 may protect the heart following ischemia-reperfusion by decreasing TNF- $\alpha$ levels (15), which has been associated with cardiomyocyte autophagy induced by LPS. In the present study, cardiomyocyte autophagy was induced by LPS, as is consistent with the results of Yuan et al (4). LDH in the serum was increased and the cell vitality was decreased following LPS treatment. This suggested that LPS may be harmful to cardiomyocytes by inducing autophagy. When the cardiomyocytes were treated with E2 $1 \mathrm{~h}$ prior to LPS, the autophagy level and LDH in the serum were attenuated and the cell vitality was increased. Therefore, E2 may protect cardiomyocytes by attenuating autophagy against LPS, mediated by the ER $\alpha$ subtype receptor.

In conclusion, the results demonstrated that E2 has an important protective role against LPS-induced injury by regulating autophagy. However, further studies are required to investigate the mechanisms underlying the interaction between E2 and the regulation of autophagy.

\section{Acknowledgements}

The present study was supported by the Nature Science Foundation of Science and Technology Commission of Shanghai municipality (grant nos. 12ZR1438000 and 12ZR1454600) and the National Nature Science Foundation of China (grant no. 81200181$)$.

\section{References}

1. Rudiger A and Singer M: The heart in sepsis: from basic mechanisms to clinical management. Curr Vasc Pharmacol 11: 187-195, 2013.

2. Zhang $\mathrm{M}$, Wang $\mathrm{X}$, Wang $\mathrm{X}$, et al: Oxymatrine protects against myocardial injury via inhibition of JAK2/STAT3 signaling in rat septic shock. Mol Med Rep 7: 1293-1299, 2013.

3. Hickson-Bick DL, Jones C and Buja LM: Stimulation of mitochondrial biogenesis and autophagy by lipopolysaccharide in the neonatal rat cardiomyocyte protects against programmed cell death. J Mol Cell Cardiol 44: 411-418, 2008.

4. Yuan H, Perry CN, Huang C, et al: LPS-induced autophagy is mediated by oxidative signaling in cardiomyocytes and is associated with cytoprotection. Am J Physiol Heart Circ Physiol 296: H470-H479, 2009.

5. Smeding L,Plötz FB,Lamberts RR, van der Laarse WJ,Kneyber MC and Groeneveld AB: Mechanical ventilation with high tidal volumes attenuates myocardial dysfunction by decreasing cardiac edema in a rat model of LPS-induced peritonitis. Respir Res 13: 23, 2012.

6. Tien YC, Lin JY, Lai CH, et al: Carthamus tinctorius L. prevents LPS-induced TNFalpha signaling activation and cell apoptosis through JNK1/2-NFkappaB pathway inhibition in H9c2 cardiomyoblast cells. J Ethnopharmacol 130: 505-513, 2010.

7. Lorne E, Dupont H and Abraham E: Toll-like receptors 2 and 4: initiators of non-septic inflammation in critical care medicine? Intensive Care Med 36: 1826-1835, 2010.

8. Leon CG, Tory R, Jia J, Sivak O and Wasan KM: Discovery and development of toll-like receptor 4 (TLR4) antagonists: a new paradigm for treating sepsis and other diseases. Pharm Res 25: 1751-1761, 2008.

9. Turdi S, Han X, Huff AF, et al: Cardiac-specific overexpression of catalase attenuates lipopolysaccharide-induced myocardial contractile dysfunction: role of autophagy. Free Radic Biol Med 53: $1327-1338,2012$.

10. Meléndez A, Tallóczy Z, Seaman M, Eskelinen EL, Hall DH and Levine B: Autophagy genes are essential for dauer development and life-span extension in C. elegans. Science 301: 1387-1391, 2003.

11. Stromhaug PE and Klionsky DJ: Approaching the molecular mechanism of autophagy. Traffic 2: 524-531, 2001.
12. Otto GP, Wu MY, Kazgan N, Anderson OR and Kessin RH: Macroautophagy is required for multicellular development of the social amoeba Dictyostelium discoideum. J Biol Chem 278: 17636-17645, 2003.

13. Vitale C, Mendelsohn ME and Rosano GM: Gender differences in the cardiovascular effect of sex hormones. Nat Rev Cardiol 6: 532-542, 2009.

14. Wenger NK: Clinical characteristics of coronary heart disease in women: emphasis on gender differences. Cardiovasc Res 53: 558-567, 2002 .

15. Xu Y, Arenas IA, Armstrong SJ,Plahta WC, Xu H and Davidge ST: Estrogen improves cardiac recovery after ischemia/reperfusion by decreasing tumor necrosis factor-alpha. Cardiovasc Res 69: 836-844, 2006

16. Jian X, Xiao-yan Z, Bin H, et al: MiR-204 regulate cardiomyocyte autophagy induced by hypoxia-reoxygenation through LC3-II. Int J Cardiol 148: 110-112, 2011.

17. Yue Z, Jin S, Yang C, Levine AJ and Heintz N: Beclin 1, an autophagy gene essential for early embryonic development, is a haploinsufficient tumor suppressor. Proc Natl Acad Sci USA 100: 15077-15082, 2003.

18. Song X, Zhang X, Wang X, et al: Tumor suppressor gene PDCD4 negatively regulates autophagy by inhibiting the expression of autophagy-related gene ATG5. Autophagy 9: 743-755, 2013.

19. Nishida K, Kyoi S, Yamaguchi O, Sadoshima J and Otsu K: The role of autophagy in the heart. Cell Death Differ 16: 31-38, 2009.

20. Wu MJ, Wang L, Ding HY, Weng CY and Yen JH: Glossogyne tenuifolia acts to inhibit inflammatory mediator production in a macrophage cell line by downregulating LPS-induced NF-kappa B. J Biomed Sci 11: 186-199, 2004.

21. Hou RC, Chen YS, Chen CH, Chen YH and Jeng KC: Protective effect of 1,2,4-benzenetriol on LPS-induced NO production by BV2 microglial cells. J Biomed Sci 13: 89-99, 2006.

22. He B, Xiao J, Ren AJ, et al: Role of miR-1 and miR-133a in myocardial ischemic postconditioning. J Biomed Sci 18: 22, 2011.

23. Whelan RS, Kaplinskiy V and Kitsis RN: Cell death in the pathogenesis of heart disease: mechanisms and significance. Annu Rev Physiol 72: 19-44, 2010.

24. Matsui Y, Takagi H, Qu X, et al: Distinct roles of autophagy in the heart during ischemia and reperfusion: roles of AMP-activated protein kinase and Beclin 1 in mediating autophagy. Circ Res 100: 914-922, 2007.

25. Wong A, Grubb DR, Cooley N, Luo J and Woodcock EA: Regulation of autophagy in cardiomyocytes by $\operatorname{Ins}(1,4,5) \mathrm{P}(3)$ and IP(3)-receptors. J Mol Cell Cardiol 54: 19-24, 2013.

26. Gurusamy $\mathrm{N}$ and Das DK: Is autophagy a double-edged sword for the heart? Acta Physiol Hung 96: 267-276, 2009.

27. Martinet W, Agostinis P, Vanhoecke B, Dewaele M and De Meyer GR: Autophagy in disease: a double-edged sword with therapeutic potential. Clin Sci (Lond) 116: 697-712, 2009.

28. Gottlieb RA and Mentzer RM: Autophagy during cardiac stress: joys and frustrations of autophagy. Annu Rev Physiol 72: 45-59, 2010.

29. Gottlieb RA, Finley KD and Mentzer RM Jr: Cardioprotection requires taking out the trash. Basic Res Cardiol 104: 169-180, 2009.

30. Deschamps AM, Murphy E and Sun J: Estrogen receptor activation and cardioprotection in ischemia reperfusion injury. Trends Cardiovasc Med 20: 73-78, 2010.

31. Booth EA and Lucchesi BR: Estrogen-mediated protection in myocardial ischemia-reperfusion injury. Cardiovasc Toxicol 8: 101-113, 2008.

32. Nikolic I, Liu D, Bell JA, Collins J, Steenbergen C and Murphy E: Treatment with an estrogen receptor-beta-selective agonist is cardioprotective. J Mol Cell Cardiol 42: 769-780, 2007.

33. Straface E, Vona R, Gambardella L, et al: Cell sex determines anoikis resistance in vascular smooth muscle cells. FEBS Lett 583: 3448-3454, 2009.

34. Jeanes HL, Tabor C, Black D, Ederveen A and Gray GA: Oestrogen-mediated cardioprotection following ischaemia and reperfusion is mimicked by an oestrogen receptor (ER) alpha agonist and unaffected by an ER beta antagonist. J Endocrinol 197: 493-501, 2008.

35. Lizotte E, Grandy SA, Tremblay A, Allen BG and Fiset C: Expression, distribution and regulation of sex steroid hormone receptors in mouse heart. Cell Physiol Biochem 23: 75-86, 2009.

36. Booth EA, Obeid NR and Lucchesi BR: Activation of estrogen receptor-alpha protects the in vivo rabbit heart from ischemia-reperfusion injury. Am J Physiol Heart Circ Physiol 289: H2039-H2047, 2005. 